\title{
An Efficient FDTD Method to Calculate Lightning Electromagnetic Fields Over Irregular Terrain Adopting the Moving Computational Domain Technique
}

\author{
Wenhao Hou ${ }^{\circledR}$, Mohammad Azadifar ${ }^{\circledR}$, Marcos Rubinstein ${ }^{\circledR}$, Fellow, IEEE, Qilin Zhang ${ }^{(\circledR}$, \\ and Farhad Rachidi ${ }^{\circledR}$, Fellow, IEEE
}

\begin{abstract}
We present an improved finite-difference timedomain (FDTD) method based on the moving computational domain technique for the calculation of lightning-generated fields over large-scale irregular terrain. In the improved method, a spatial window moving along with the wavefront at the speed of light is defined and, at each time step, the field components are updated only in that window. The time delay of the wavefront arrival caused by the propagation effect and the irregular terrain is considered when determining the width of the moving window. The efficiency and accuracy of the improved model are validated by comparing its results with those calculated by conventional FDTD, considering an example of lightning-radiated electromagnetic field propagation over complex mountainous terrain. The gain in the CPU time can be as high as a factor of 4 to 5 , while the accuracy remains unchanged with respect to the conventional FDTD.
\end{abstract}

Index Terms-Arrival time delay, finite-difference time-domain (FDTD) efficiency, irregular terrain, lightning electromagnetic field, moving computational domain.

Manuscript received March 8, 2019; revised April 30, 2019; accepted May 11,2019 . This work was supported in part by the National Key R\&D Program of China under Grant 2017YFC1501505, in part by the National Natural Science Foundation of China under Grants 41775006 and 41575004 , and in part by the scholarship from the China Scholarship Council. (Corresponding author: Farhad Rachidi.)

W. Hou is with the Key Laboratory of Meteorological Disaster, Ministry of Education (KLME)/Collaborative Innovation Center on Forecast and Evaluation of Meteorological Disaster/Key Laboratory for Aerosol-Cloud-Precipitation of China Meteorological Administration, Nanjing University of Information Science and Technology, Nanjing 210044, China, and also with the Electromagnetic Compatibility Laboratory, Swiss Federal Institute of Technology, Lausanne 1015, Switzerland (e-mail: hwhnuist@163.com).

M. Azadifar and F. Rachidi are with the Electromagnetic Compatibility Laboratory, Swiss Federal Institute of Technology, Lausanne 1015, Switzerland (e-mail: mohammad.azadifar@epfl.ch; farhad.rachidi@epfl.ch).

M. Rubinstein is with the University of Applied Sciences Western Switzerland, Yverdon-les-Bains 1401, Switzerland (e-mail: marcos.rubinstein@ @eigvd.ch).

Q. Zhang is with the Key Laboratory of Meteorological Disaster, Ministry of Education (KLME)/Collaborative Innovation Center on Forecast and Evaluation of Meteorological Disaster/Key Laboratory for Aerosol-Cloud-Precipitation of China Meteorological Administration, Nanjing University of Information Science and Technology, Nanjing 210044, China (e-mail: zhangqilin71@163.com).

This paper has supplementary downloadable material available at http://ieeexplore.ieee.org, provided by the author.

Color versions of one or more of the figures in this paper are available online at http://ieeexplore.ieee.org.

Digital Object Identifier 10.1109/TEMC.2019.2917282

\section{INTRODUCTION}

I $\mathrm{N}$ RECENT years, interest in the fast evaluation of lightning fields over long distances has steadily increased, including studies on the propagation effects along irregular terrain and the interaction between lightning electromagnetic fields and the lower ionosphere (e.g., [1]-[3]). The finite-difference timedomain (FDTD) technique has become a common method used in these studies, since it is relatively easy to include a complex terrain, as well as an inhomogeneous soil and ionospheric parameters.

The FDTD computation burden and simulation time increase quickly with the increase of the simulation domain size. Several approaches to handle this problem have been proposed, including the use of a moving window FDTD (e.g., [4], [5]), the segmented long-path propagation technique [6], and the use of parallel computing (e.g., [7], [8]). All these approaches are efficient and can reduce the memory requirements associated with FDTD. However, their implementation is not always straightforward.

In order to improve the efficiency of FDTD for the calculation of the very low frequency (VLF) and low frequency (LF) wave propagation over thousands of kilometers in the earthionosphere waveguide, Bérenger proposed a moving computational domain technique (MCDT) [9], [10] that only updates the field components within a reduced domain at each time step. In this letter, we adapt the MCDT in the FDTD method for the calculation of lightning-generated groundwave propagation over irregular terrain. A minimum moving domain/window which can satisfy the computational requirement is defined, and its effectiveness for the propagation over irregular terrain is analyzed. The gain in computation time after adopting the MCDT is also estimated. Finally, the improved FDTD method with MCDT is applied to study the propagation effect of lightningradiated fields along complex mountainous terrain around the Säntis Mountain, and the efficiency and accuracy of the model are verified.

\section{DESCRIPTION OF THE METHOD}

\section{A. Moving Computational Domain Technique}

The two-dimensional FDTD (2-D FDTD) model in cylindrical coordinate systems has been widely used for evaluating 


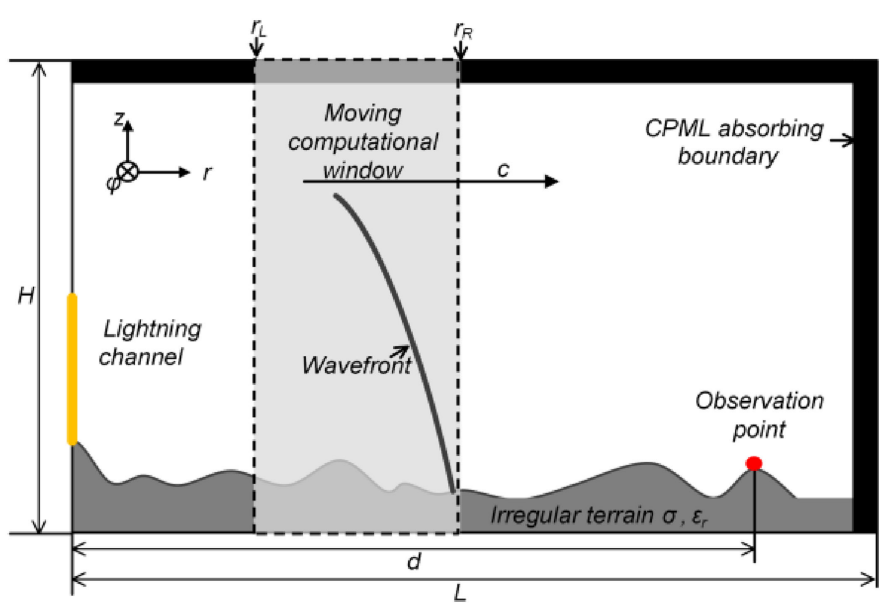

Fig. 1. Configuration of the 2-D FDTD model with the MCDT. The lightning channel is placed on the axis of symmetry. The dashed rectangle defines the window which moves along with the wavefront at the speed of light $c$. Only the field components in this spatial window are updated at each time step. The soil is characterized by a finite conductivity $\sigma$ and a relative permittivity $\varepsilon_{r} . d$ is the horizontal distance from the lightning channel to the observation point, shown by the red dot.

the lightning electromagnetic fields over irregular terrain. Fig. 1 shows the overall configuration of the FDTD model for lightning groundwave propagation in the low frequency band. The lightning channel is placed on the symmetry axis, and the upper and right boundaries are surrounded by a convolutional perfectly matched layer (CPML) absorbing boundary [11]. The bottom boundary is usually either a perfect electrical conductor flat ground or irregular terrain with finite conductivity $\sigma$ and relative permittivity $\varepsilon_{r}$. In addition, the conformal FDTD method [12] is usually used to improve the calculation accuracy of the field near the nonflat surface.

Here, we focus on the fields generated by the lightning discharge at distances of tens to several hundreds of kilometers. Since we are usually only interested in the field waveform within a time window of some tens of microseconds after the wave propagates to the observation point, we introduce a method based on the MCDT [9], [10] to improve the FDTD calculation efficiency.

According to the propagation properties of the electromagnetic wave, the electromagnetic energy within a considered time window distributes within a corresponding window in the spatial domain. As a result, only the field components in this domain/window need to be updated at every time step. The largest propagation velocity of the wavefront is the speed of light $c$. Therefore, the right/leading border of the window is defined as

$$
r_{R}(t)=\min [c t, L]
$$

where $t$ is the present time in FDTD and $L$ is the width of the whole FDTD domain. In practical applications, considering the effectiveness of the absorbing boundary, it is suggested to expand the right border by several grid size units. Since we are only interested in the field waveform within $t_{w}$ after the wave has reached the observation point, the left/trailing border of the window can be defined as

$$
r_{L}(t)=\max \left[0, c\left(t-t_{w}\right)\right]
$$

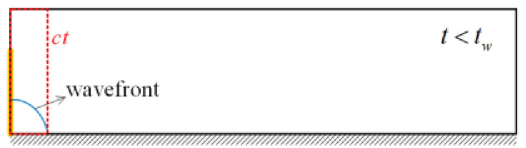

(a)

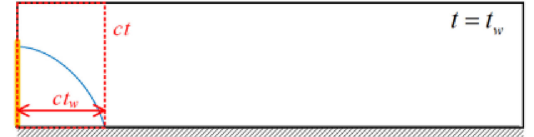

(b)

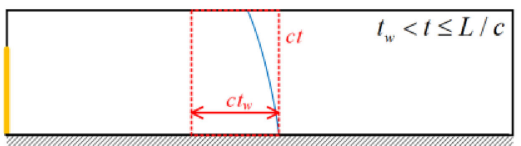

(c)

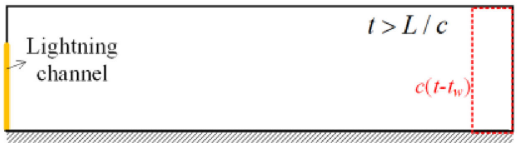

(d)

Fig. 2. Illustration of the MCDT. The red dashed rectangle shows the position of the computational domain/window. Only the field components in the window are updated at every time step. $t$ is the present iteration time in FDTD, $t_{w}$ is the considered time window, and $c$ is the speed of light. The thickness of absorbing boundary is not shown in this illustration.

in which the time window $t_{w}$ is defined by the users according to their specific requirements. It should be noted, however, that when the lightning electromagnetic wave propagates over a finitely-conducting terrain, or when the observation point is not located on the ground surface, the arrival time of the wavefront at the observation point will be delayed compared to $d / c$ ( $d$ being the horizontal distance between the lightning channel base and the observation point). Therefore, the left border defined in (2) should be at least extended to

$$
r_{L}(t)=\max \left[0, c\left(t-t_{w}-t_{\text {delay }}\right)\right]
$$

where $t_{\text {delay }}$ is the arrival-time delay of the wavefront compared to $d / c$, accounting for the height of the observation point, the propagation effect over the finitely-conducting ground, and the irregular terrain along the propagation path.

Fig. 2 illustrates the MCDT for the case of a lightning discharge striking a flat ground. When the present time $t$ is smaller than the time window $t_{w}$, the width of the moving window increases as the time increases [see Fig. 2(a)]. When $t_{w} \leq t \leq L / c$ [see Fig. 2(c)], the width of the moving window is $c t_{w}$ and the complete window moves at the speed of light $c$. After the leading border of the window has reached the right boundary of the whole FDTD domain, the width of the moving-window begins to decrease [see Fig. 2(d)].

It is very important to make sure that all the electromagnetic energy which can be received at the observation points within the time window $t_{w}$ is included in the considered spatial window. Fig. 3 gives an illustration of regions where the electromagnetic energy can propagate to a given observation point (A) within the considered time window $t_{w}$. Assume the wavefront arrives at the point $\mathrm{A}$ at $t_{\text {arrival }}$. Note that the right border of the window at $t_{\text {arrival }}$ may be ahead of the wavefront because of the time delay 


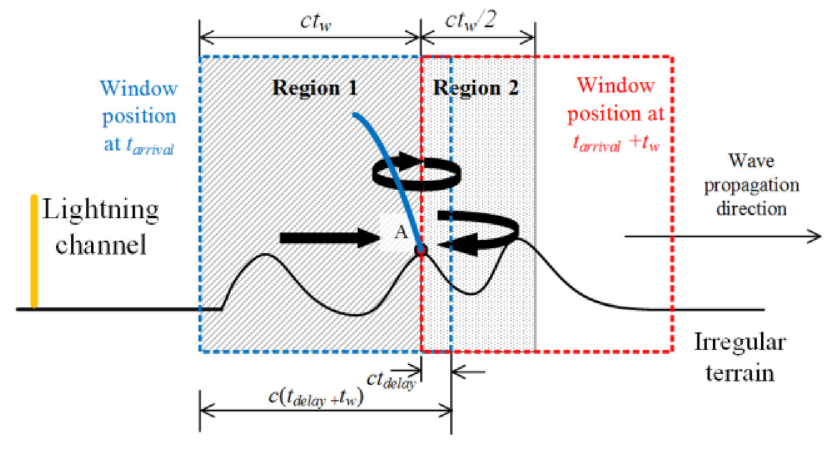

Fig. 3. Illustration of regions where the electromagnetic energy can propagate to the observation point $\mathrm{A}$ within the considered time window $t_{w}$. Assume the wavefront reached the observation point $\mathrm{A}$ at $t_{\text {arrival }}$. Region 1 defines the upstream region where the forward-propagating wave energy can propagate to A within $t_{w}$. Region 2 defines the downstream region where the back-scattered wave energy can propagate to $\mathrm{A}$ within $t_{w}$.

caused by the irregular terrain (see the blue dashed rectangle in Fig. 3). Region 1 defines the largest upstream region where the forward-propagating wave can reach the observation point A within the time $t_{w}$. Region 2 defines the largest downstream region where the back-scattered wave can reach A within $t_{w}$. At time $t_{\text {arrival }}+t_{w}$, the forward-propagating wave from the left border of region 1 and the scattered wave from the right border of region 2 reach simultaneously the observation point A. Note that regions 1 and 2 are defined by assuming wave propagation at the speed of light $(c)$; actually, since the propagation speed is slightly lower than $c$, the corresponding regions are slightly smaller than regions 1 and 2. However, this does not have an influence on the accuracy of our method. In addition, the multiple reflections that can be observed at A within $t_{w}$ can also be included in the spatial window. Therefore, all the electromagnetic energy that can be received at the observation point $\mathrm{A}$ within $t_{w}$ will be included in the proposed moving window. The accuracy of the method will be validated in the following section.

\section{B. Estimation of the Parameter $t_{\text {delay }}$}

In this section, we present a method to quickly estimate the parameter $t_{\text {delay }}$ for the case of lightning-generated electromagnetic pulse propagation over a long-range complex terrain, in which the propagation effect of the finite ground conductivity and the irregular terrain should be taken into account. The arrival time delay caused by the propagation effect can be approximately calculated using

$$
t_{\text {delay }}=0.0046 d+0.8073
$$

or

$$
t_{\text {delay }}=0.0033 d+0.1461
$$

where $d$ is the propagation distance in $\mathrm{km}$, and $t_{\text {delay }}$ is the propagation delay in $\mu$ s. Equations (4) and (5) are the linear fitting results for the typical first return stroke and subsequent return stroke, respectively (see the supporting document for details). In addition, the "terrain envelope method" [1] can be used to evaluate the additional arrival time delay introduced by the irregular terrain.

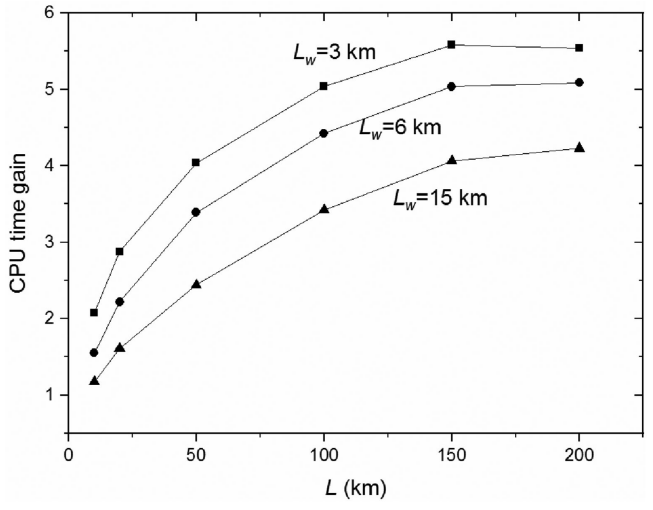

Fig. 4. CPU time gain of the improved method based on the MCDT relative to the conventional FDTD model for different moving window widths $\left(L_{w}\right)$ and widths of the FDTD simulation domain $(L)$. Three different moving window widths of 3,6 , and $15 \mathrm{~km}$ were considered, and the corresponding values of $t_{w}$ were 10,20 , and $50 \mu \mathrm{s}$ when $t_{\text {delay }}$ equals zero, respectively. The simulations were executed on the Intel Xeon E5-1650 processor at $3.2 \mathrm{GHz}$.

\section{Gain in Computation Time}

In the improved method based on MCDT, since only a portion of the field components needs to be updated at each time step, the computational efficiency will be improved compared to the conventional FDTD approach which requires an update of all the field components in the whole FDTD domain. The gain is directly (although not linearly) dependent on the width of the moving window $\left(L_{w}\right)$ and the width of the whole FDTD domain $(L)$. The smaller either of these parameters is, the shorter the computation time.

To illustrate the effectiveness of the new method, different spatial window sizes and simulation domain sizes were considered. The height of the FDTD simulation domain $(H)$ was set to $10 \mathrm{~km}$, the grid size and the time step were set, respectively, to $\Delta r=\Delta z=10 \mathrm{~m}$ and $\Delta t=16.6 \mathrm{~ns}$. A 15 -layer CPML absorbing boundary was adopted, and the corresponding thickness $\delta_{\text {cpml }}$ was set to $150 \mathrm{~m}$. The observation point was located on the ground surface and the horizontal distance from the lightning channel to the observation point was $d=L-\delta_{\mathrm{cpml}}$. The total number of iteration timesteps for each case is given by $n=\left(d / c+L_{w} / c\right) / \Delta t$. Fig. 4 shows the gain in the computation time as a function of $L$, which has been defined as

$$
C P U \text { Time Gain }=\frac{\text { CPU time for model without MCDT }}{\text { CPU time for model with MCDT }} \text {. }
$$

It can be seen from Fig. 4 that the use of the proposed method based on the MCDT can greatly increase the FDTD efficiency in terms of computation time. The bigger the FDTD domain, the higher the achieved gain, and the gain can be as high as a factor of 4 to 5 when the width of the simulation space is larger than $150 \mathrm{~km}$. More discussion on the computational efficiency can be found in the supporting document.

\section{APPLICATION}

In this section, we will apply the improved method to the study of the lightning electromagnetic wave propagation over a long, complex terrain around the Säntis Mountain in 


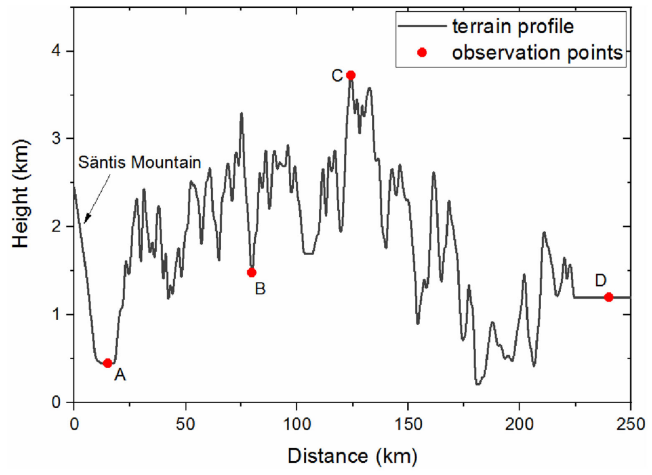

Fig. 5. 2-D terrain profile along the electromagnetic wave propagation path. The red dots show the positions of observation points.

TABLE I

TOTAL CPU TIME FOR THE IMPROVED MODEL WITH MCDT AND THE MODEL WITHOUT USING MCDT

\begin{tabular}{ccc}
\hline \hline Method & $\begin{array}{c}\text { Total iteration } \\
\text { timesteps }\end{array}$ & $\begin{array}{c}\text { Total CPU } \\
\text { time }\end{array}$ \\
\hline FDTD method without using MCDT & 49400 & $27.9 \mathrm{~h}$ \\
FDTD method using MCDT & 49400 & $5.3 \mathrm{~h}$ \\
\hline \hline
\end{tabular}

Northeastern Switzerland. The accuracy of the proposed model will also be validated by comparing its results to those obtained using a conventional FDTD approach.

Fig. 5 shows the 2-D terrain profile (assuming axial symmetry) from the Säntis tower along a path directed southeast (the same path was also considered in [1]). The profile was obtained from the global digital elevation model version 2 (GDEM V2) data measured from the advanced spaceborne thermal emission and reflection radiometer.

The conductivity of the terrain (ground and mountain) was set to $0.001 \mathrm{~S} / \mathrm{m}$, and the relative permittivity was set to 10 . The length of the lightning channel was set to $H_{c h}=7.5 \mathrm{~km}$, and the return stroke velocity was set to $v=150 \mathrm{~m} / \mu \mathrm{s}$. A typical subsequent return stroke channel-base current was used [13], with a peak value of $12 \mathrm{kA}$ and a maximum steepness of $40 \mathrm{kA} / \mu \mathrm{s}$. The modified transmission line model with exponential decay [14], [15] assuming a current decay constant $\lambda=2 \mathrm{~km}$ was adopted [16]. The grid size was $\Delta \mathrm{r}=\Delta \mathrm{z}=10 \mathrm{~m}$, and the time step was $\Delta \mathrm{t}=16.6 \mathrm{~ns}$. The simulation domain was $245 \mathrm{~km}$ long and $10 \mathrm{~km}$ high. The red dots in Fig. 5 show the positions of four observation points. The horizontal distances between the lightning channel and the considered observation points are 15,80 , 124 and $240 \mathrm{~km}$, respectively.

The time window $t_{w}$ was set to $20 \mu \mathrm{s}$, and the corresponding width of the spatial window was set to $7 \mathrm{~km}$, which was extended by $1 \mathrm{~km}$ considering the arrival-time delay of the wave when propagating in the mountainous area. Table I shows the total CPU time for the improved FDTD method with MCDT as well as that without using MCDT. It can be seen that, for the same total iteration timesteps, the total CPU time adopting the MCDT was reduced by a factor of 5 , from $27.9 \mathrm{~h}$ down to $5.3 \mathrm{~h}$, which is consistent with the predicted results from Fig. 4.

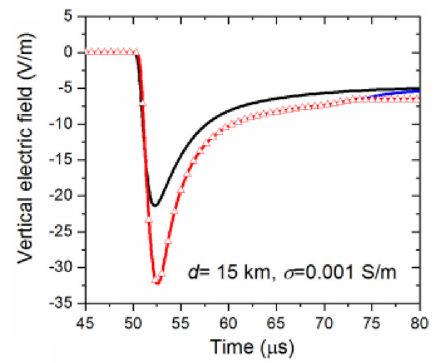

(a)

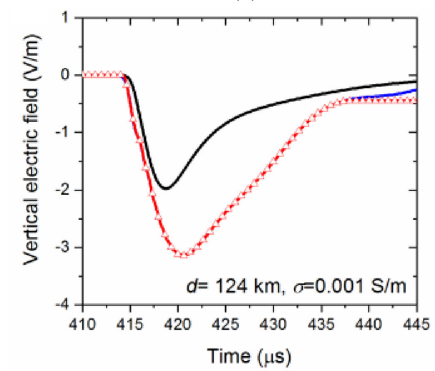

(c)

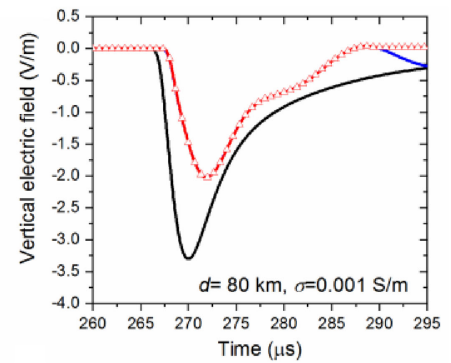

(b)

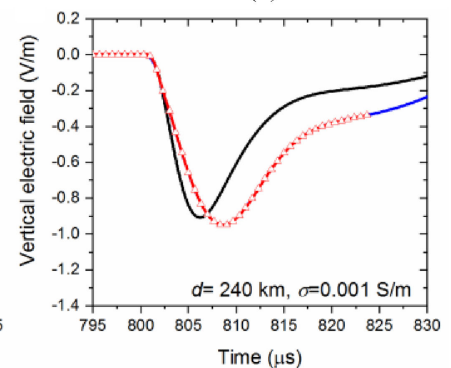

(d)
- flat ground $\quad$ real terrain,conventional FDTD $\quad-$ - real terrain,FDTD with MCDT

Fig. 6. Vertical electric field at four observation points calculated by the improved model with MCDT (red lines) and the results without adopting MCDT (blue lines). The black lines show the results for lightning striking flat ground. For the results calculated by adopting MCDT, as expected, the calculated electric field value stays constant after the initial $20 \mu$ s time window.

Fig. 6 shows the vertical electric field at the considered observation points calculated by the FDTD method with MCDT and the results obtained using a conventional FDTD method. For the sake of comparison, the fields corresponding to the case of a flat ground are also presented in the figures. The propagation process over mountainous terrain is complex, with forwardpropagation, reflection, scattering, and diffraction. However, the waveforms calculated using MCDT overlap with those for the conventional FDTD approach, thus confirming the accuracy of the new method.

It can be seen from Fig. 6, that the vertical electric field on the ground surface can be affected differently by the complex terrain profile, depending on the observation point. The reasons for the observed effects have been discussed in [1] and [17]. In summary, the enhancement of the fields for observation points A and $\mathrm{C}$ is mainly due to the reflection processes in the transition between the mountain and the flat ground [17]. In addition, the observation point $\mathrm{B}$ being in a valley, the field is largely reduced compared to a flat ground. It is also interesting to note that, for observation point $\mathrm{B}$, due to the elongated propagation path [1], the wave arrival time for the terrain is delayed by about $1.5 \mu \mathrm{s}$ compared to the case of a flat ground. On the other hand, for observation point $\mathrm{C}$, the wave arrival time for the complex terrain is ahead of that for a flat ground.

\section{SUMMARY}

We presented an improved FDTD method based on the MCDT for the calculation of lightning-generated fields over largescale irregular terrain. In the proposed method, a spatial window moving along with the wavefront at the speed of light is 
defined, considering the wave arrival time delay introduced by the propagation effect and the irregular terrain. At each time step, the field components are updated only in that window.

The efficiency and accuracy of the improved method were validated by comparing its results with those calculated by conventional FDTD, considering an example of lightning-radiated electromagnetic fields propagating over complex mountainous terrain. We showed that the gain in the CPU time can be as high as a factor of 4 to 5, while the accuracy remains unchanged with respect to the conventional FDTD. The method can be straightforwardly extended to a 3-D domain.

\section{REFERENCES}

[1] D. Li, M. Rubinstein, F. Rachidi, G. Diendorfer, W. Schulz, and G. Lu, "Location accuracy evaluation of ToA-based lightning location systems over mountainous terrain," J. Geophys. Res., vol. 122, pp. 11760-11775, 2017.

[2] M. Azadifar et al., "Analysis of lightning-ionosphere interaction using simultaneous records of source current and $380 \mathrm{~km}$ distant electric field," J. Atmos. Solar-Terr. Phys., vol. 159, pp. 48-56, Jun. 2017.

[3] W. Hu and S. A. Cummer, "An FDTD model for low and high altitude lightning-generated EM fields," IEEE Trans. Antennas Propag., vol. 54, no. 5, pp. 1513-1522, May 2007.

[4] B. Fidel, E. Heyman, R. Kastner, and R. W. Ziolkowski, "A hybrid rayFDTD moving frame approach to pulse propagation," J. Comput. Phys., vol. 138, no. 2, pp. 480-500, 1997.

[5] R. J. Luebbers, J. W. Schuster, and K. C. Wu, "Full wave propagation model based on moving window FDTD," in Proc. Mil. Commun. Conf., vol. 2, 2003, pp. 1397-1401.

[6] M. W. Chevalier and U. S. Inan, "A technique for efficiently modeling propagation for use in both FDFD and FDTD," IEEE Antennas Wireless Propag. Lett., vol. 5, no. 1, pp. 525-528, Dec. 2006.
[7] T. Oikawa, J. Sonoda, M. Sato, N. Honma, and Y. Ikegawa, "Analysis of lightning electromagnetic field on large-scale terrain model using threedimensional MW-FDTD parallel computation," Elect. Eng. Jpn., vol. 184, no. 2, pp. 20-27, 2013.

[8] G. G. Pyrialakos, T. T. Zygiridis, N. V. Kantartzis, and T. D. Tsiboukis, "GPU-based calculation of lightning-generated electromagnetic fields in 3-D problems with statistically defined uncertainties," IEEE Trans. Electromagn. Compat., vol. 57, no. 6, pp. 1556-1567, Dec. 2015.

[9] J. P. Berenger, "FDTD computation of VLF-LF propagation in the earthionosphere waveguide," Ann. Telecommun., vol. 57, no. 11-12, pp. 10591090, 2002.

[10] J. P. Berenger, "Long-range propagation of lightning pulses using the FDTD method," IEEE Trans. Electromagn. Compat., vol. 47, no. 4, pp. 1008-1012, Nov. 2005.

[11] J. A. Roden and S. D. Gedney, "Convolution PML (CPML): An efficient FDTD implementation of the CFS-PML for arbitrary media," Microw. Opt. Technol. Lett., vol. 27, no. 5, pp. 334-339, 2000.

[12] W. Yu and R. Mittra, "A conformal finite difference time domain technique for modeling curved dielectric surfaces," IEEE Microw. Wireless Compon., vol. 11, no. 1, pp. 25-27, Jan. 2001.

[13] F. Rachidi et al., "Current and electromagnetic field associated with lightning return strokes to tall towers," IEEE Trans. Electromagn. Compat., vol. 43 , no. 3, pp. 356-367, Aug. 2001.

[14] C. A. Nucci, C. Mazzetti, F. Rachidi, and M. Ianoz, "On lightning return stroke models for LEMP calculations," in Proc. 19th Int. Conf. Lightning Protection, Apr. 1988.

[15] F. Rachidi and C. A. Nucci, "On the master, Lin, Uman, Standler and the modified transmission line lightning return stroke current models," $J$. Geophys. Res., vol. 95, pp. 20 389-20 394, Nov. 1990.

[16] C. A. Nucci and F. Rachidi, "Experimental validation of a modification to the transmission line model for LEMP calculations," in Proc. 8th Int. Symp. Electromagn. Compat., Mar. 7-9, 1989, pp. 389-394.

[17] W. Hou, Q. Zhang, L. Wang, and J. Zhang, "Effect of striking a cone-shaped mountain top on the far lightning-radiated electromagnetic field," IEEE Trans. Electromagn. Compat., early access, 2018, doi: 10.1109/TEMC.2018.2843772. 